\title{
Colliers de perles. Transmission, circulation, mémoire du féminin
}

Pearl necklaces. Feminine gender memory, transmission, circulation

Patrizia Ciambelli

\section{(2) OpenEdition}

12 Journals

Édition électronique

URL : https://journals.openedition.org/tc/6554

DOI : $10.4000 /$ tc. 6554

ISSN : 1952-420X

Éditeur

Éditions de l'EHESS

\section{Édition imprimée}

Date de publication : 15 décembre 2012

Pagination : 78-95

ISBN : 978-2-7351-1534-1

ISSN : 0248-6016

\section{Référence électronique}

Patrizia Ciambelli, «Colliers de perles. Transmission, circulation, mémoire du féminin », Techniques \& Culture [En ligne], 59 | 2012, mis en ligne le 15 décembre 2015, consulté le 29 septembre 2022. URL: http://journals.openedition.org/tc/6554; DOI : https://doi.org/10.4000/tc.6554 


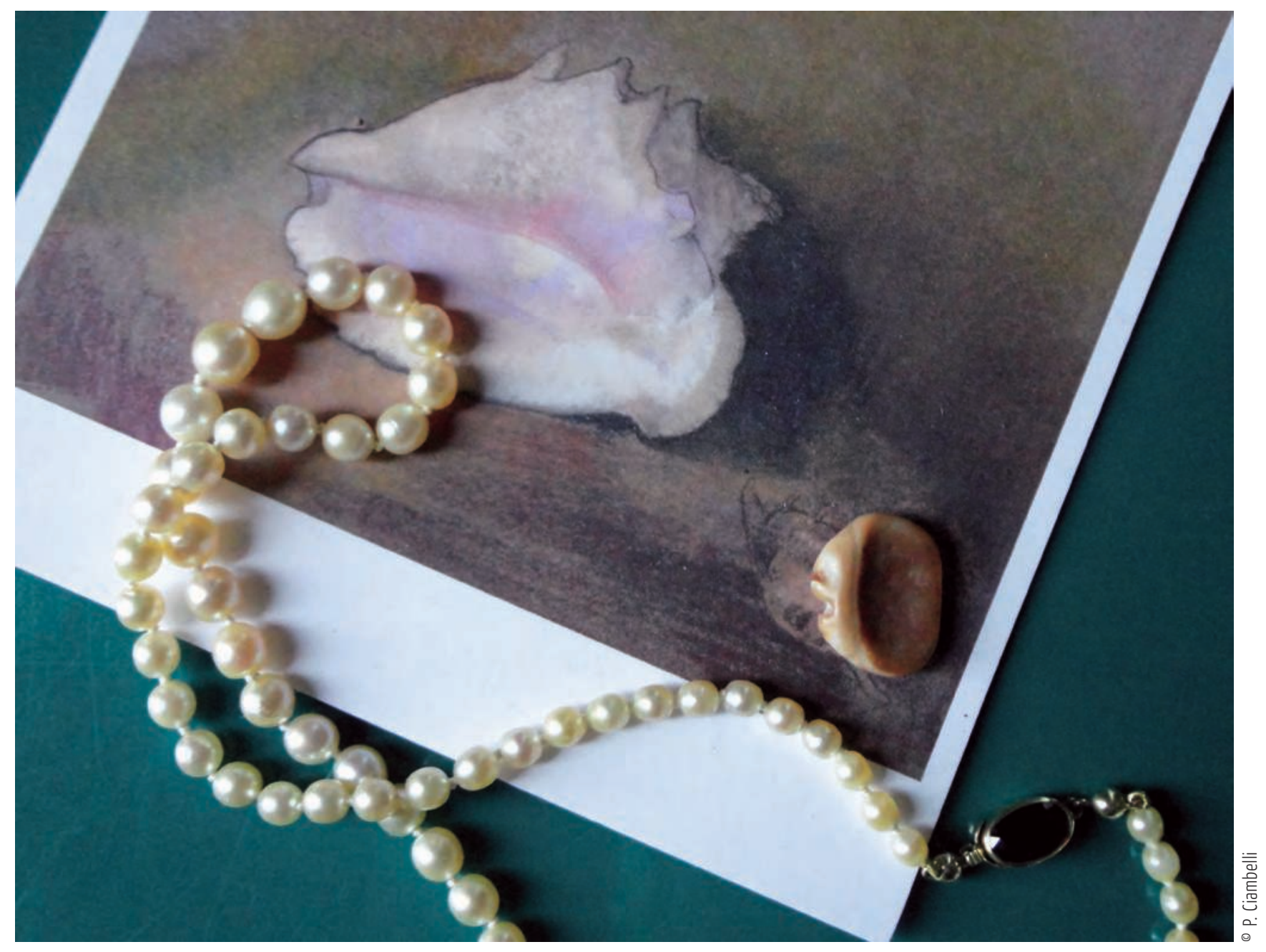




\section{Patrizia Ciambelli}

Centre d'Anthropologie Sociale (LISST)

patriciamb@club-internet.fr
Itinéraires de coquillages - 1

Techniques \& Culture 59, 2012/2 : 78-95

\section{COLLIERS DE PERLES}

\section{Transmission, circulation, mémoire du féminin}

Lorsqu'on les nomme au pluriel, les perles désignent d'emblée le collier. Loin d'être relégué au statut de bijou vieillot, le collier de perles se réaffirme aujourd'hui pour tous les âges et tous les styles.

Certes les codes de l'apparence et les règles d'usage sont remis en question voire inversés, détournés, contournés. De très longs sautoirs s’allongent sur la poitrine et le dos à toutes les heures du jour et de la nuit, doubles et triples fils d'énormes perles côtoient le classique dégradé de perles blanches longtemps délaissé tout comme la paire en boucles d'oreille. Entre ces deux extrêmes, une multitude de variantes reviennent en force tant dans la bijouterie de fantaisie que dans la joaillerie. Il n'en est pas moins que la perle, marque esthétique par définition de la jeune fille en fleur, de la mariée et de la femme accomplie, plus que jamais en ce moment s'impose ${ }^{1}$.

Vraies et fausses, "de nature" et "de culture", rondes et baroques, d'eau douce et d'eau salée, claires et foncées, les perles sont convoitées tant par des jeunes femmes en attente du "beau bijou" pour leurs dix-huit ans, que par des hommes en quête de cadeaux surprenants, ou encore par des femmes indépendantes voulant affirmer leur singularité et leur autonomie.

Cette matière-parure qui désigne à plein titre l'univers du féminin investit également les produits cosmétiques, le maquillage et participe de l'esthétique vestimentaire. La ligne perlière épouse, souligne, dessine les formes féminines ou alors agit en trompe l'œil se servant comme autrefois de la « semence de grains » qu'on vendait au poids et avec laquelle on réalisait le « perlage » pour apporter de la brillance et du décor aux vêtements qui en étaient en même temps rigidifiés et alourdis. À ces miniatures font écho les grosses perles 
artificielles qui soulignent de façon ostentatoire les contours d'une veste ou qui viennent s'imprimer sur les tissus mêmes. Toute la gamme des accessoires sert aussi de support aux décorations perlières: chapeaux, sacs, barrettes pour les cheveux, chaussures. Les lunettes de soleil Chanel avec une perle enfilée dans chaque tige se distinguent par leur élégance et rappellent une fois de plus la passion vouée par la créatrice de mode aux perles inséparables de son parcours professionnel et personnel.

Le corps-bijou, tendance de la mode d'aujourd'hui avec les

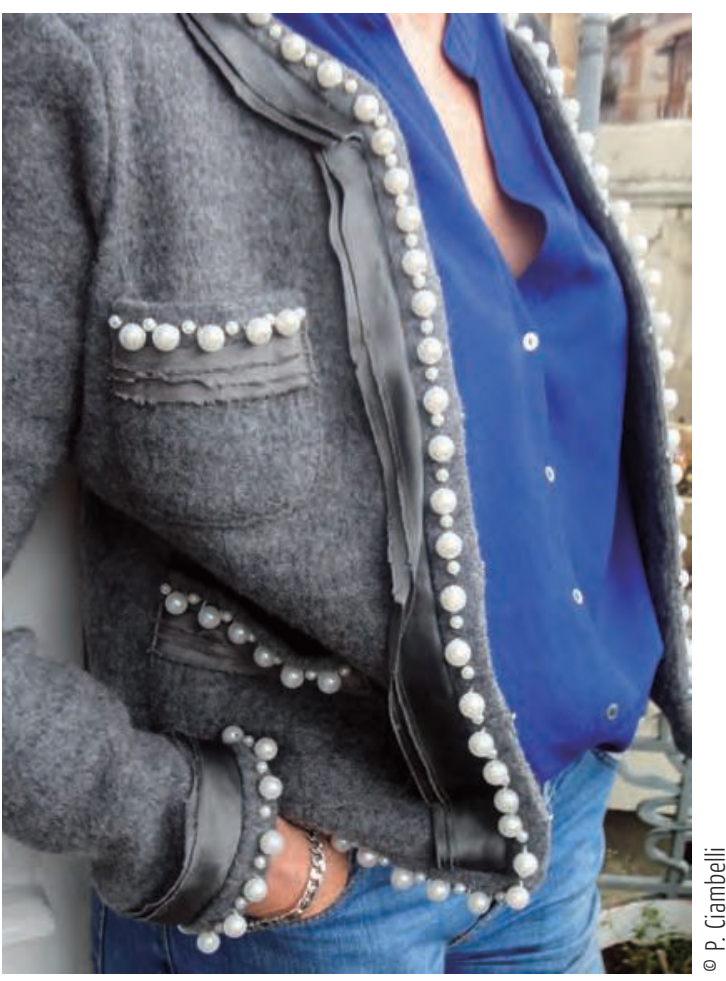

La ligne perlière

Comme autrefois,

la perle participe de nos jours aussi de l'esthétique vestimentaire: ici le « perlage » marque tout le contour d'une veste en apportant du poids et de la brillance. parures qui ont investi le corps dans son intégralité, devient luimême corps perlé avec ses cache-cœurs, bustiers et même des robes réalisées entièrement en perles blanches. Dans une société où la question du genre est au premier plan, il est intéressant qu'une matière associée si intimement à l'univers du féminin s'impose et décidément du côté des femmes ${ }^{2}$. On peut penser que cet accueil si favorable qui leur est réservé s'accorde aussi avec une certaine éthique environnementale de nos temps qui a fait récemment la fortune d'autres matières « naturelles » aux vertus prophylactiques, telles par exemple le corail ou l'ambre pour les colliers de dentition des enfants ${ }^{3}$.

La redécouverte et la revalorisation de cette " matière première » sont lisibles même dans les vitrines des bijoutiers où elle a regagné un espace de visibilité: perles du Japon et de Chine s'opposent à celles des mers du sud et de Tahiti, évocation exotique d'un « orient » indissociable de la perle et qui constitue avec le lustre un de ses caractères essentiels pour qualifier sa brillance, sa luminosité, sa valeur. Certains créateurs de bijoux contemporains ont relancé aussi les perles de rivière de petite taille et bien nacrées en les associant à l'argent ou à des matières ordinaires mais « naturelles » comme le caoutchouc. Leurs réalisations jouent sur le contraste chromatique du noir et blanc et sur le métissage matériel et culturel en s'inspirant des modèles de parures exotiques.

La marque TS - Trésor Secret - spécialiste en France des créations en perles de culture compte plusieurs boutiques dans les principales villes outre des espaces duty-free dans les aéroports du monde entier garantissant l'authenticité de la matière et du travail artisanal des bijoutiers adhérents. Leur clientèle est donc très hétérogène d'autant plus que les premiers prix sont abordables et les styles très différents, des classiques aux plus « tendances » comme les gros colliers où se mélangent des perles du même diamètre très irisées aux nuances vert, bleu, aubergine. Le vocabulaire qui désigne leurs collections s'approprie le lexique de la perle: Très Or, Un jour ailleurs, Au pays des Merveilles, Opposition, Étoile dans le Ciel, Belle composition, Moi c'est encore moi!, Japonaise. Leur site mis en ligne illustre les variétés et qualités, les conseils pour l'entretien ainsi que l'histoire des perles et le légendaire de leur origine ${ }^{4}$. Car, comme le réaffirme la propriétaire d'une autre importante bijouterie toulousaine, pour vendre les perles il faut d'abord en raconter l'histoire. 


\section{À L'ORIGINE}

D'où elles viennent, comment elles se forment, combien de variétés existent, comment on les choisit pour les accorder à son désir, à sa personne. "On doit expliquer qu'il faut beaucoup de temps pour la formation de la perle et surtout faire comprendre que chaque perle est unique ». Unio, sans pareille, ainsi l'appelaient les Romains qui en faisaient un usage immodéré sur le corps, les vêtements ainsi que les statues des divinités et les symboles du pouvoir impérial, ce qui suscita un jugement moral très sévère et positionna la margarita du côté de l'excès et de l'ostentation.

Emprunté au grec margaritês, le mot latin pour dire la perle est resté dans l'usage du prénom Marguerite ${ }^{5}$ qui désigne également la fleur dont les filles interrogent les pétales pour connaître l'amour. Mais il est également doublé par celui de Perle qui a donné lieu à un champ lexical extrêmement diversifié dans les domaines culinaire, architectural, médical, musical et dont le sens au figuré peut être valorisant (une personne aux qualités extraordinaires, une parfaite dentition) ou inversement dépréciatif (une voix de petite perle, lâcher une perle, enfiler des perles) ${ }^{6}$; le très célèbre verset de la Bible (Matthieu VII, 6) « jeter des perles aux cochons » est passé désormais dans le langage courant.

Les perles font donc leur première apparition dans la vie féminine à travers l'attribution du prénom qui inscrit sous le signe de la parure et qui instaure la première analogie entre la perle et le féminin. À l'image de la Vénus de Botticelli, emblème de la beauté naissante, représentée dans sa nudité comme la perle au milieu du coquillage, la femme est identifiée à la matière précieuse - blanche, ronde, polie, lumineuse. Mais cette analogie préfigure en même temps son destin de génitrice tout comme l'animal qui engendre la perle enrobant progressivement d'épaisseurs de nacre l'infime corps étranger pénétré accidentellement (ou bien introduit volontairement) dans le coquillage, représentation qui prime encore dans notre imaginaire depuis l'Antiquité. La peinture de la Renaissance en donne à voir quelques exemples. Ainsi La pêche des perles d'Alessandro Allori (1535-1607), réalisé pour le « studiolo » de François I de Médicis. Une foule de personnages - hommes et femmes avec nymphes, tritons et jolis « putti », les corps nus entremêlés, le regard tourné vers les bateaux des pêcheurs -, exhibent au premier plan une profusion de perles ruisselant de gros coquillages traités avec un souci naturaliste et présentés ouverts, en guise de récipients remplis de perles, comme des écrins précieux.

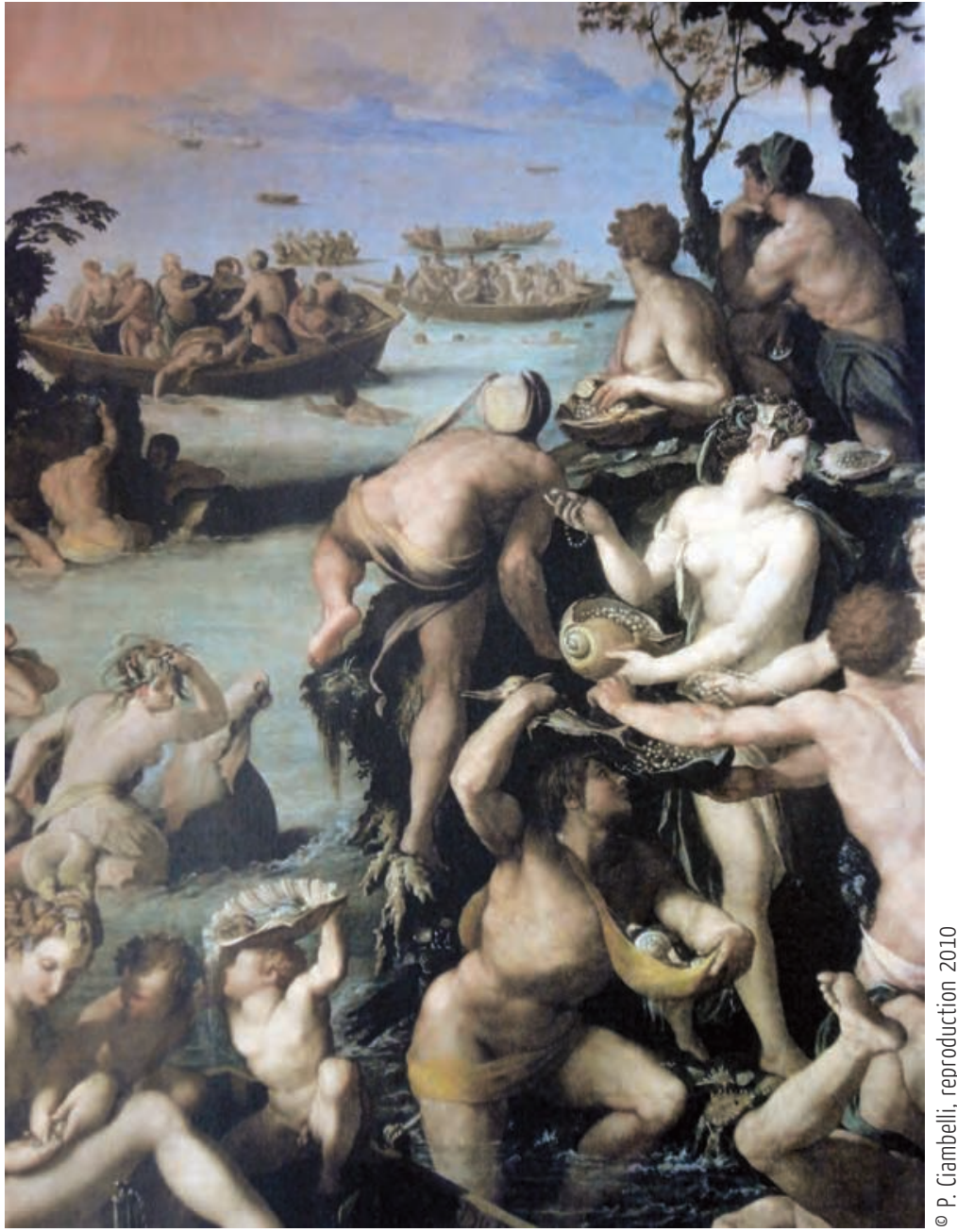

\section{La cueillette merveilleuse (Alessandro Allori, La pesca delle perle v.1570, Florence, Palazzo Vecchio)}

Une multitude de personnages masculins et féminins avec nymphes, tritons et jolis putti, saisie lors d'une cueillette frénétique dans une profusion de perles qui ruissellent de gros coquillages. 


Femmes aux perles
(Jacopo Zucchi,
Allégorie de la découverte
de l'Amérique, vers 1585, Rome
Galleria Borghese. Détail)
À la fois immergées au sein
d'un monde sous-marin comme
remonté à la surface,
es figures féminines de toute beauté
arborent les trésors sous forme de
bijoux, de perles et de coquillages.
Le thème de la beauté naissant de
l'eau trouve son paradigme
dans le célèbre tableau de Botticelli
dans lequel Vénus s'offre de même
comme la perle au sein du coquillage.

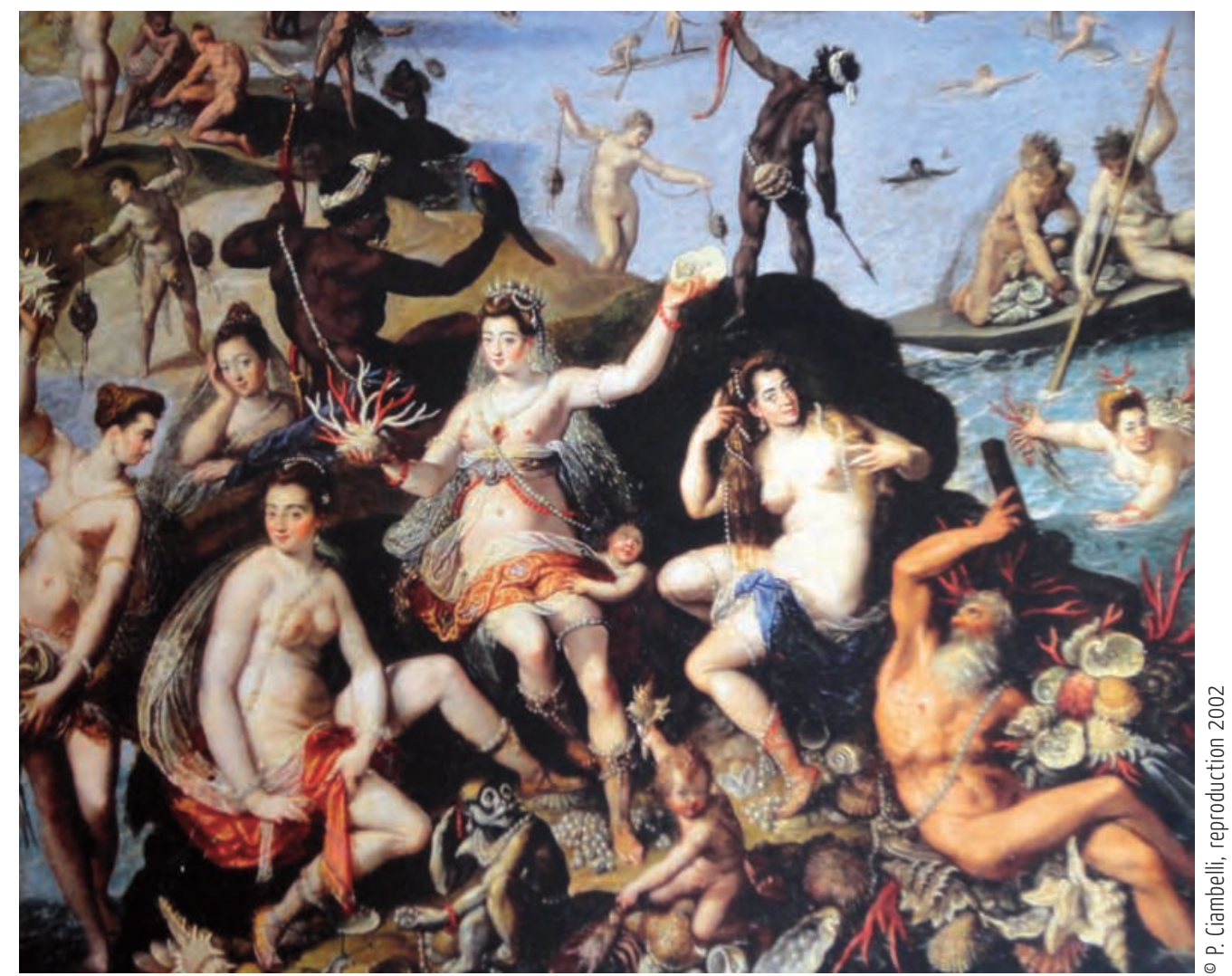

Dans l'Allégorie de la découverte de l'Amérique de Jacopo Zucchi (vers 1585) des figures féminines de toute beauté arborent ces trésors du monde sous-marin remontés à la surface dans leurs formes naturelles - coquillages ouverts et perles en quantité à leurs pieds - ainsi qu'exhibés comme parures: colliers, sautoirs en bandoulière, bracelets, couronnes, ceintures, fils lovés dans les cheveux, tissus transparents décorés de perles. Femmes et perles participent de cet univers aquatique qui semble donner une origine aux unes et aux autres et qui recèle nombre de mystères et curiosités de la nature: trésors enfouis, divinités et êtres fantastiques. D’autres analogies sont lisibles dans cette représentation: la blancheur, la brillance, la noblesse, la rareté mais aussi la prolificité, la beauté, la séduction? ${ }^{7}$.

Le monde des profondeurs nous renvoie également au mythe d'origine de la perle selon lequel la coquille remonte des profondeurs de la mer à la surface à la première heure du matin et en baillant, absorbe la rosée et le rayonnement du soleil, de la lune et des étoiles ce qui lui fait engendrer la perle lui donnant plus ou moins de blancheur et de luminosité.

Pline précise dans son Histoire naturelle (IX, LIV):

«Si la rosée est pure le produit est blanc, si elle est trouble le produit est terne; il est pâle s'il a été conçu à l'approche d'un orage; ce qui prouve que l'état des perles dépend plus du calme des airs que du calme des mers. C'est du ciel qu'elles tirent une couleur nuageuse ou limpide, suivant la sérénité des matinées ». 
Cette croyance étiologique attestée depuis l'Antiquité et reprise ensuite au Moyen Âge montre donc que la perle est le produit d'une transmutation de l'état liquide à l'état solide, même si le durcissement des «petites pierres blanches et précieuses » se produit seulement une fois remontées à la surface par les pêcheurs et sorties de leurs coquillages. La cueillette est donc une condition du processus d'achèvement de formation de la perle et de son entrée dans le monde humain ${ }^{8}$.

Le mythe d'origine se complète du récit légendaire de la pêche à la perle à l'aide de l'agate qui, comme attesté dans les lapidaires médiévaux, aurait des vertus prophylactiques et thérapeutiques parmi lesquelles celles de "régler» les femmes et protéger des mauvais sorts et des envoûtements. Accrochée à une cordelette solide que les pêcheurs font descendre dans la mer, l'agate attirée par la perle, lorsqu'elle la trouve, s'immobilise et la corde ne bouge plus ${ }^{9}$.

Une autre transmutation - cette fois des humeurs - concerne les larmes et renvoie à un autre mythe d'origine ${ }^{10}$ : les larmes d'Ève chassée du paradis terrestre tombant dans la mer se changèrent en perles tandis que celles tombées sur la terre produisirent les plus belles fleurs. Larmes de genèse qu'on retrouve dans une position inversée dans la croyance selon laquelle offrir des perles porte malheur - il faut les acheter soi-même ou les transmettre - et cause les larmes, ce qui instaure une analogie entre la perle et l'oeil ${ }^{11}$.

Dans les contes merveilleux ce sont les mots prononcés par la bouche d'une héroine « de toute beauté » qui sortent en forme de perles, don extraordinaire d'une fée en échange de sa générosité et modestie, alors que sa sœur ou demi-sœur, laide et mauvaise, est condamnée à cracher des crapauds et des serpents ${ }^{12}$.

Le corps de la femme est ici comme le coquillage qui engendre les perles et qui dans le temps continue de les nourrir à travers le contact de sa peau, surface de frontière et d'échange entre le visible et l'intime, le caché et le montré. Privée de ce contact corporel, on dit que la perle perd sa brillance, s'opacifie, "se ride » jusqu'à jaunir, c'est-à-dire à mourir, donc il faut porter un collier pour être belle mais aussi pour entretenir sa beauté. Mais l'inverse est aussi vrai et les « humeurs » féminines peuvent les ternir dans certaines conditions.

Dans ces jeux d'échanges de propriétés entre la matière première et le corps des femmes s'énonce, en effet, ce qu'est la «nature » féminine ${ }^{13}$. Une nature excessive, toujours à régler, à corriger, à stabiliser par des objets esthétiques qui fonctionnent à la fois comme opérateurs d'identité et marques visibles d'un statut acquis selon les âges de la vie.

Or, l'apprentissage de la beauté à travers le collier de perles, objet emblématique du monde de la parure féminine, se fait à partir de la première enfance à travers une fascination corporelle qui oscille entre le regard à distance et le contact lorsqu'on se l'autorise. Ce parcours initiatique où la personne à qui le bijou appartient joue un rôle déterminant, s'accompagne aussi d'une parole, d'un récit, d'une généalogie familiale qui se transmet avec elle et qui constitue le fondement d'une mémoire destinée à circuler. Tout au long de ce parcours, des « perles » apparaissent sous formes différentes pour les premiers jeux d'embellissement. 


\section{PERLES D'ENFANCE}

\section{Premières parures}

La première dent de lait en pendentif témoigne de l'analogie avec la perle qui se réaffirme aussi par la Dentale: un petit coquillage utilisé depuis la préhistoire comme parure et monnaie d'échange. Bien que délicat et fragile, sa forme allongée très pointue évoquant une corne, et naturellement percée, facilite son usage en collier.
Les premières perles «naturelles » en guise de parures s'inscrivent dans un processus de croissance avec la poussée des dents de lait accompagnée souvent de " percées " douloureuses que les jeunes parents essaient de soulager et de favoriser en mettant au cou de l'enfant un bijou, le collier d'ambre ${ }^{14}$.

Au fur et à mesure que les dents tombent, les enfants les cachent sous l'oreiller dans l'espoir que la petite souris vienne les chercher pendant la nuit laissant en échange une pièce ou une étrenne. Ces « perles » en principe sont perdues à jamais alors que ceux qui peut-être ignorent cette coutume les conservent dans une petite boîte à dents.

Mais la première dent «perdue » peut être traitée de façon singulière et changer de statut, devenant alors un objet de collecte, lorsqu'elle est déposée avec d'autres marques de la première fois - le bracelet de naissance, le cordon ombilical, le premier bijou, la première mèche de cheveux. Ou bien devenir bijou, lorsqu'elle est transformée en pendentif et suspendue au bracelet de la fillette avec d'autres porte-bonheur - la corne en or, le cochon en corail, la perle dans son coquillage - qui s'ajoutaient autrefois, d'anniversaire en anniversaire. Petit bijou-talisman qui n'est pas sans évoquer certaines parures des sociétés lointaines réalisées avec les parties dures du corps humain et animal censées transmettre au porteur les qualités de la matière dure et impérissable ${ }^{15}$.

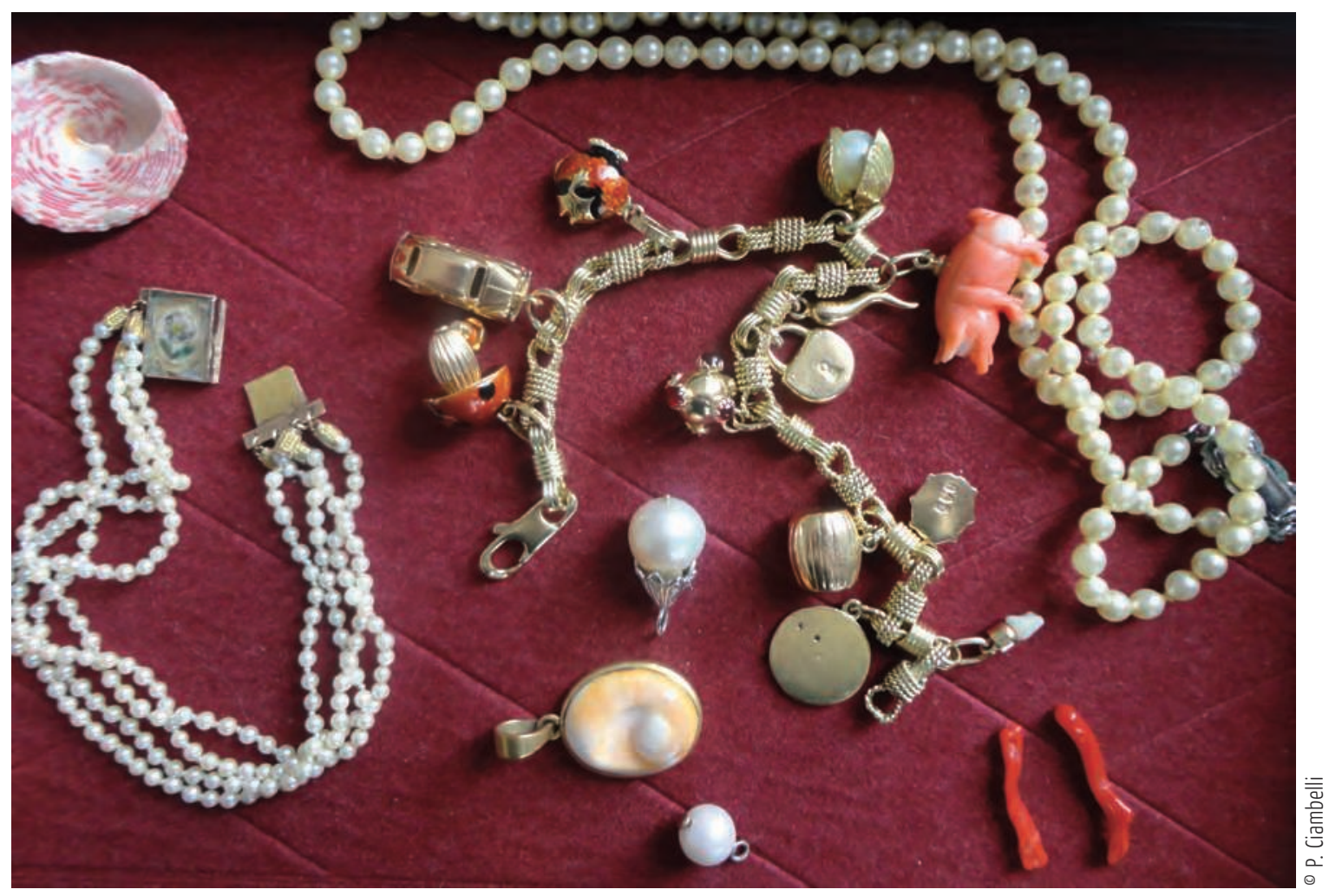


C'est dans l'univers de la nature que l'on saisit des perles éphémères avec lesquelles toute fillette a pu expérimenter les premières fabrications de collier: ainsi, les pâquerettes qu'on enfile et s'amuse à porter en guise de collier avec les classiques cerises en boucles d'oreilles avant de les manger. Mais il y a aussi les colliers et bracelets que les petites filles enfilent elles-mêmes se servant de perles artificielles en pâte de verre, en bois, en plastique, aujourd'hui déclinées dans toutes les formes, couleurs, imitations et variantes culturelles.

Les billes avec leur multitude de couleurs, de tailles, de formes, transparentes ou opaques, brillantes ou iridescentes sont plutôt les perles des garçons qui, à la différence de celles des filles, ne sont pas percées ${ }^{16}$.

L'espace domestique recèle d'autres parures qui s'apparentent à l'univers perlier: par exemple, dans la boîte à ouvrage ${ }^{17}$ où logent les boutons en nacre - la madreperla, la mère de la perle - dont on se sert pour toute une gamme d'objets personnels et de décor: bijoux, coupelles, nécessaires de toilette, éventails, flacons de parfum, jumelles de théâtre, hochets, etc. Mais les enfants aiment aussi aller découvrir d'autres trésors de la maison: les « vrais bijoux » abrités dans les coffrets ou dans les tiroirs de la chambre à coucher des parents. Les jeux d'initiation à la coquetterie et à la séduction peuvent se faire en cachette ou sous la conduite d'une fillette un peu plus âgée qui encourage quelques « essais » de colliers ou même grâce à la complicité d'une grand-mère qui ouvre à la fois son coffret à bijoux et son armoire pour permettre à la petite-fille de se déguiser en « dame ». L'interdit de toucher posé par les femmes qui détiennent les bijoux n'est pas toujours respecté.

« Un après-midi d'été, avec ma sœur aînée nous avions décidé de nous déguiser avec les chapeaux et les habits de notre mère. C'était notre jeu préféré. Nous essayions aussi les parfums, le maquillage, tout sauf les bijoux que nous avions le droit juste de regarder, mais pas de toucher. Ce jour-là, en ouvrant l'étui en velours rouge où les trois rangs de perles du collier de maman dessinaient comme une vague, la tentation avait été trop forte. Lorsqu'on avait quitté la chambre, sans me faire remarquer par ma sœur, j'avais pris le collier laissant l'étui et je m'étais renfermée dans ma chambre. Je ne sais plus combien de temps j'avais passé à le caresser, à le serpenter sur le couvre-lit et puis je l'avais enfin mis autour du cou mais juste posé, sans l'attacher, en imaginant le jour qu'il serait à moi. Rêve impossible, car je savais qu'il était destiné déjà à ma sœur. En le remettant à sa place, je me souviens encore que je tremblais de peur que ma mère - et encore plus ma sœur - me découvre».

Comme toute initiation, l'accès aux bijoux est ressenti comme la découverte d'un pan de l'existence que l'on devine mais dans lequel on ne peut encore pénétrer, d'un « secret » qu'il faut dérober. Secret d'un monde féminin, d'un monde sensuel dont font partie aussi les parfums et leurs flacons, les pierres et leurs formes, le maquillage et ses couleurs. À travers ce souvenir d'enfance on voit aussi se profiler le rôle joué par les perles convoitées par la sœur cadette exclue de la destination du bijou à cause du rang de naissance.

Lapprentissage passe aussi par les gestes qu'on s'autorise et qu'on garde pour toujours, comme celui de passer son doigt dans le collier et enrouler les perles autour. Lorsqu'elle est dans une situation d'anxiété, Nicole répète ce même geste qu'elle faisait petite en jouant avec le collier de sa grand-mère et qui l'apaise tout comme autrefois quand elle était dans les bras de son aieule. Anne raconte que parfois sa grand-mère l'embrassait si fort, que les perles lui faisaient mal mais elle n'osait rien dire par crainte de lui faire de la peine. C'est comme ça aussi qu'elle a appris que les perles changent de température: elle les sent devenir chaudes, d'autres fois fraîches ou carrément froides. Valérie se rappelle qu'avec son frère, ils jouaient à compter les perles du collier que leur grand-mère portait en permanence en partant de la 


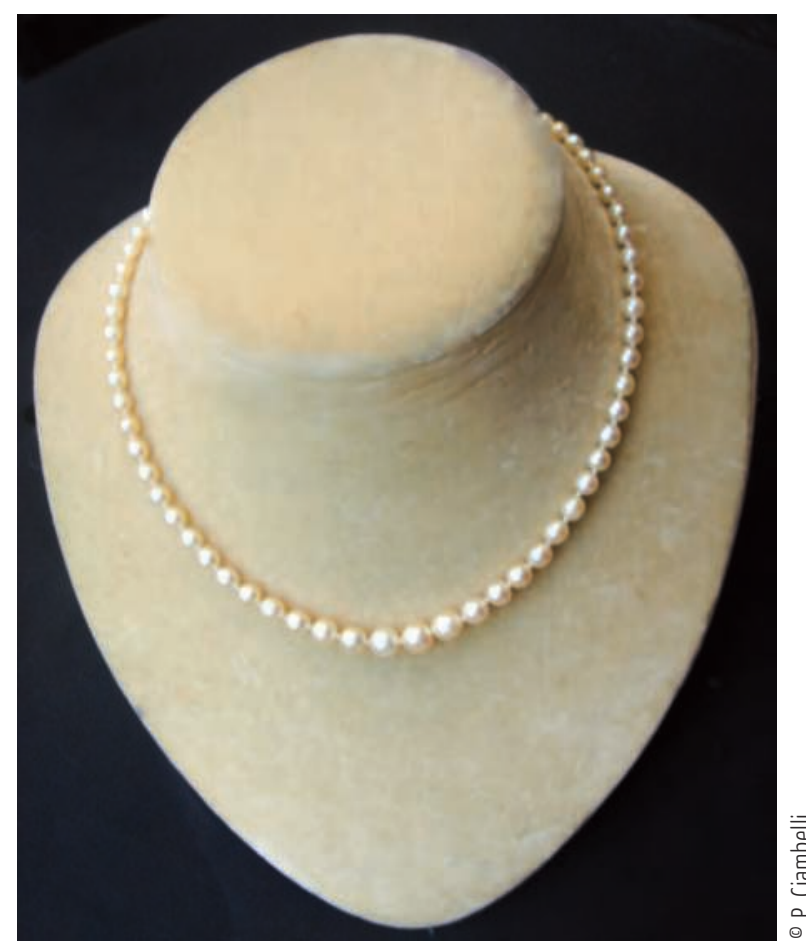

Perles du Japon

Évocation exotique d'un « Orient » indissociable, le fil de « blanches » en dégradé qui a été longtemps le bijou féminin incontournable se réaffirme aujourd'hui pour tous les âges et tous les styles renversant les codes de l'apparence et les règles d'usage. plus grosse au milieu jusqu'à la plus petite accrochée au fermoir faisant chacun le demi-tour du collier.

« Pour nous c'était un peu comme des billes qu'on faisait rouler contre sa peau, parfois elle nous grondait pour nos gestes brusques qui mettaient à dure épreuve les nouds du collier ${ }^{18}$.

Des bijoux délicats avec de toutes petites perles fines sont offerts aux fillettes sous la forme de bracelets ou de colliers légers avec juste une « belle » perle à peine un peu plus grosse au milieu. Mais c'est plutôt lorsque commence le « temps des amours », qu'on acquiert le droit d'en posséder et surtout de les porter et de les exhiber sur soi. Catalina qui a aujourd'hui cinquante-huit ans se souvient qu'entre douze et quinze ans, elle et ses sœurs ont reçu des colliers et des boucles d'oreille avec des perles mais c'était le collier qui marquait un passage vraiment important et significatif. « On nous offrait le collier de perles mais pour notre anniversaire, et spécialement pour les quinze ans, parce qu’on avait grandi. Ma mère les gardait tous dans la boîte à bijoux de la maison parce qu'on avait le droit de le mettre que pour les fêtes. »

Il fallait donc une occasion exceptionnelle pour que la jeune fille en fleur puisse arborer ce collier « princesse » avec la plus belle et la plus grosse perle venant marquer la frontière entre la ligne du cou et la poitrine, point d'attraction du regard extérieur et érotisation du corps féminin à travers son dévoilement ${ }^{19}$.

Ces perles de jeune fille qui viennent doter d'un pouvoir de séduction et préfigurent déjà un autre temps de la vie, se conforment au code esthétique de la parure selon une loi de progression. Aussi prend-on soin de limiter la taille des perles, la longueur du fil, la couleur pour que le collier soit « simple et discret ». Des partages d'âge s'opèrent donc en fonction des mesures, du style du collier et des couleurs. L'excès - en taille, forme, nombre - renvoie à une image de femme trop sexuée impudique, provocatrice, qui s'oppose à l'image de modestie et de pureté que les perles véhiculent simultanément.

Les « blanches » restent selon la majorité des bijoutiers les favorites des jeunes femmes à cause de leurs nuances rosées et argentées et parce qu'elles « s'accordent mieux à la peau », « font briller » et « donnent de l'éclat » alors que les plus foncées aux nuances vert, bleus, gris, aubergine seraient surtout les préférées des femmes autour de la cinquantaine. Ce qui n'empêche pas à une perle «noire » unique en pendentif dans une version très épurée sur un fil transparent de se voir destinée aux plus jeunes tout comme les plus âgées rêvent du collier de perles blanches qu'aucune aïeule ne leur a transmis.

\section{ACCORDS ET DÉSACCORDS}

« Les disgrâces souvent sont du Ciel révélées; j’ai songé cette nuit, de perles défilées... ». Ainsi dit Mascarille dans Le dépit amoureux de Molière (acte V, scène 6). 
Il arrive en effet que le bijou vienne préfigurer de façon concrète l'inscription d'une vie dans un destin. Les éclatements des colliers qui soudain se défont en éparpillant une cascade de perles et brisant le mouvement perpétuel de sa forme circulaire sont attribués à la nature propre au bijou tout comme à la nature « explosive» de certaines femmes. Pourtant, on sait à quel point le fil est soumis à l'usure du temps et au frottement contre la nacre brute qu'il traverse au cœur de la perle. L'enfilage est une opération délicate qui requiert une compétence particulière; elle est exercée aujourd'hui encore en majorité par les femmes dans un cadre domestique, tout comme autrefois. On se sert de préférence du fil en soie, à son tour matière noble, précieuse, douce et naturelle, tout comme la perle. La qualité du fil et la technique des nœuds déterminent dans le collier la finesse et la juste souplesse pour donner une belle ligne et provoquer un léger tintement, tout en évitant que les perles s'entrechoquent en se frottant et tournant sur elles-mêmes. C'est pour cette raison aussi qu'on les conserve séparément et à l'abri dans un étui, même si d'autres raisons peuvent être à l'origine de cette précaution, qui renvoient à la connotation de la perle comme un œil avec son « iris » caché. Un œil aveugle quand on la sort du coquillage, voyant lorsqu'on la perce pour la convertir en parure -, et qui peut s'avérer «mauvais » et source de malheur ${ }^{20}$.

Conséquence contrée justement par tout un système: la surface polie qui reflète et renvoie l'image, la « sonorité » du bijou, les nœuds tout le long du cercle, caractères, qui dans la tradition, assurent un rôle de gardien de la personne contre des actes négatifs potentiels.

En se brisant le collier de perles agit comme signe prémonitoire ou présage néfaste, comme dans ce souvenir de Bella Chagall dans son œuvre autobiographique (1994 : 312313). Devant la brillance et la limpidité des perles que le bijoutier lui montre, Madame Bishowskaya décide de lui confier le secret qui l'oppresse depuis longtemps. Sa mère avait un collier de perles petites et ternes qui « avaient pris sur sa peau ridée une nuance jaune », le seul héritage qui lui venait de sa propre mère, morte lorsqu'elle était encore un enfant. Ce collier, qui l'avait parée le jour de son mariage, elle le mettait chaque fois qu'elle devait accoucher - elle eut neuf enfants - persuadée qu'ainsi « venait à son chevet sa mère, morte toute jeune, et qu'elle les bénissait, elle et son nouveau-né ». Une fois, la dernière, elle eut un enfant mort: elle ne le savait pas encore mais tout son corps la démangeait, comme si une armée de fourmis était en train de la dévorer. En effet, on trouva dans son lit d'accouchée les perles de son collier qui s'était défait; elles avaient roulé en se répandant dans les draps. C'est ainsi qu'elle avait compris la perte de son enfant et qu'en même temps que le collier, sa jeunesse et sa féminité s'étaient brisées. À Madame Bishowskaya de conclure:

«Vous voyez quel est le pouvoir des perles? » ${ }^{21}$.

Plusieurs bijoutiers ont affirmé fermement que contrairement à d'autres bijoux, on ne vend jamais un collier de perles: « cela ne se fait pas ». Ce propos est en effet partagé par la majorité de mes interlocutrices ainsi que la croyance selon laquelle il ne faut jamais offrir des perles: cela porte malheur.

Il est destiné donc à être conservé par les femmes qui peuvent, voire doivent, le porter - nous avons vu à quel point pour les perles cela s'avère vital - même si le temps du profit personnel, lorsqu'on

\section{L'œil et la perle}

Dans certaines familles de la région de Forlì en Italie, on posait dans la main d'un nouveau-né une fleur ou une bague « fleurie » d'une perle pour le doter de la vertu de guérir les maux des yeux dont l'albugo ou tache blanche de la cornée appelée « perle ».

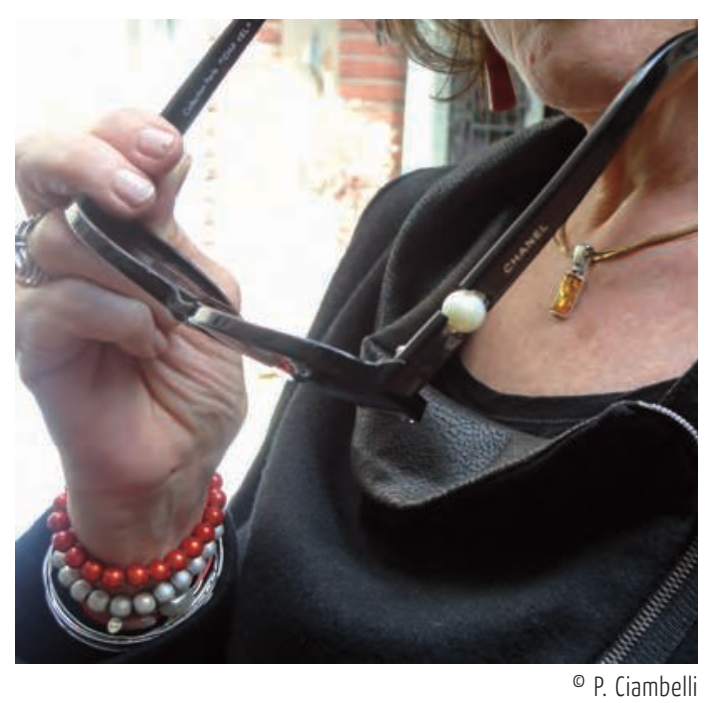




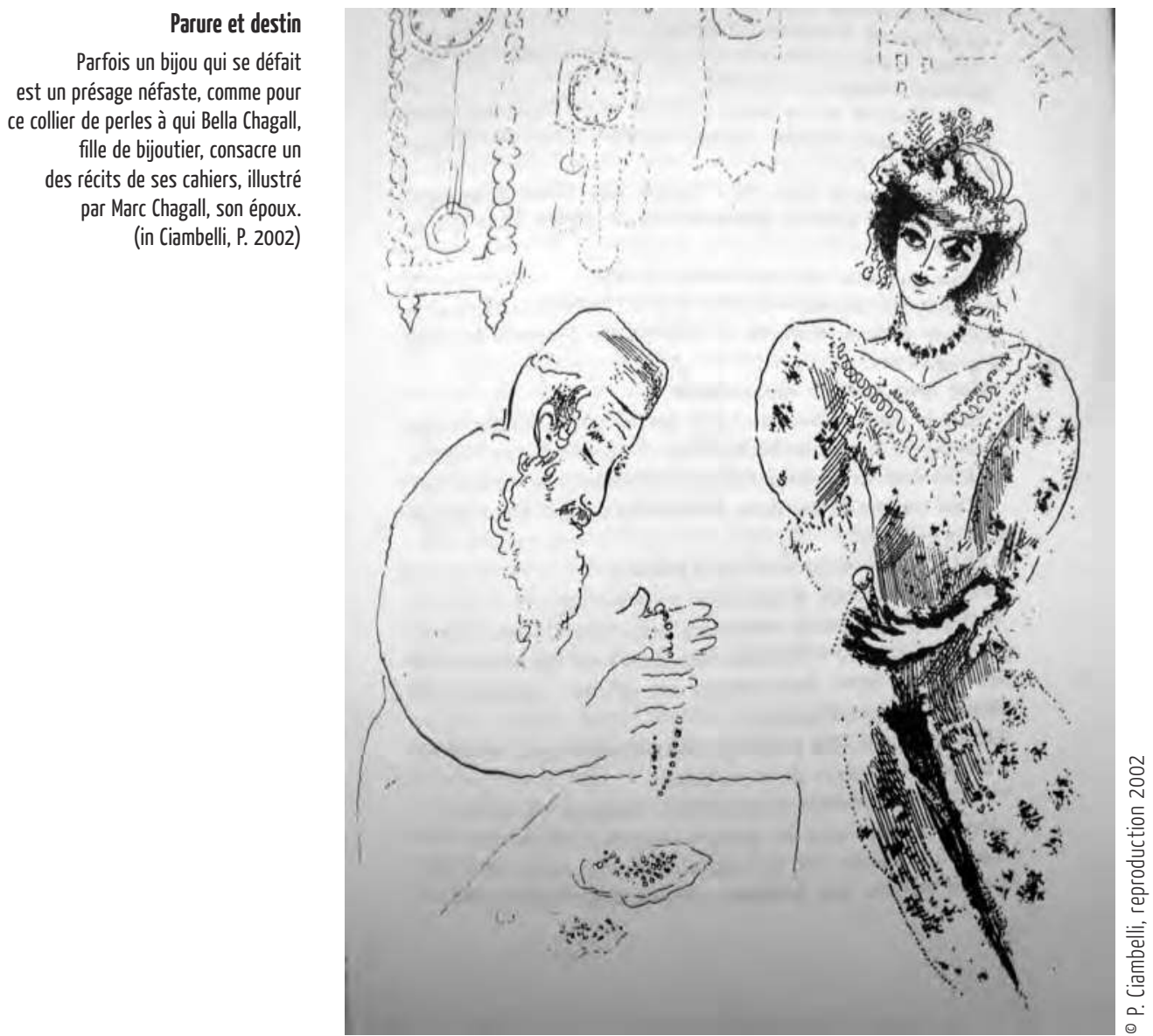

échappe aux contingences plus ou moins cruelles qui en causent la perte, est limité à un temps de la vie soumis aux règles de la beauté, de la séduction, de l'apparence.

Le posséder signifie donc l'immobiliser un temps avant de le faire circuler ensuite d'une génération à l'autre: de grand-mère à petite-fille, de mère à fille, de tante à nièce ${ }^{22}$. Ce que lui donne un statut qui l'apparente aux «bijoux de famille » même si ceux-ci restent ancrés dans une logique patrilinéaire et cela malgré l'éclatement du modèle classique du couple et de la famille avec les nouveaux cas de figure et la diversification des liens de parenté. Cependant, ce sont toujours les femmes - consanguines ou alliées - les dépositaires de ces objets précieux qu'elles devront ensuite transmettre à la personne destinée. Et c'est justement la circulation dans le temps qui va renforcer la valeur de ce « bien» impérissable, qui témoigne concrètement d'une reproduction vitale et crée les présupposés pour un temps à venir. Or, le geste de faire passer le collier de perles implique qu'il soit accompagné d'une parole qui puisse en restituer le sens et transmettre la singularité des événements qui en font son histoire. C'est ainsi qu'il peut exprimer et véhiculer la chaîne relationnelle entre les vivants et les morts et donc la continuité et le renouvellement de la généalogie familiale qui fait dire 
à une femme: "Le collier de perles est pour moi le seul lien qui me reste encore avec ma famille et la vie qui est passée ».

Transmettre n'est pas seulement laisser un souvenir, mais aussi « envoyer audelà », faire passer d'une personne à l'autre, d'un espace à l'autre, un bien nourri par un contact corporel et par un vécu personnel. La notion de souvenir revient constamment même si elle joue de façon ambivalente en raison de ce que le collier véhicule. Cela se traduit pour certaines personnes dans un attachement, parfois déraisonnable, qui fait qu'elles l'incorporent sans pouvoir plus s'en séparer, alors que d'autres, consciemment ou obscurément, le mettent à l'écart - oubli, abandon, perte - parce qu'il s'avère trop « lourd » à porter. Cette lourdeur engendre une impossibilité à la jouissance qui peut prendre la forme d'une gêne physique à le «sentir» contre sa propre peau.

« Je ne pouvais même pas imaginer de porter ces perles, elles étaient trop liées à l'image de ma mère qui les portait souvent, j'avais l'impression de la priver de quelque chose. Elle me les avait données la veille de mon mariage. J'avais mis le collier ce jour-là, mais avec le sentiment qu'il ne pouvait pas m'appartenir, d'ailleurs tout de suite après je l'avais occulté dans ma boîte à bijoux. Et puis, à sa mort, ce collier est devenu mon bijou: je ne le quitte jamais, parfois je le garde aussi en dormant tellement il fait partie de moi, d'ailleurs je me touche le cou tout le temps pour être sûre que les perles soient bien là, à leur place».

Ce quelque chose qui appartient vraiment aux femmes, c'est bien leur féminité, leur « beauté » que les perles expriment et qui fait qu'une fille ne puisse pas prendre à sa mère ce qu'elle peut accepter plus facilement de sa grand-mère. À travers la transmission de ce bien que sont les perles, une passation de pouvoir a lieu. C'est ce même pouvoir de séduction que se voit attribuer Denise, lorsque la grand-mère, après avoir parcouru d'un regard attentif à la fois ses joyaux et le visage de sa petite fille, lui destine le joli collier de perles fines cadeau d'antan de ses fiançailles.

Succéder à un proche en prenant son bijou revient à prendre sa place, d'une certaine façon à assumer son identité - d'ailleurs il n'est pas rare qu'on porte le même prénom - ce qui explique pourquoi certaines femmes considèrent ce geste par rapport à un vivant comme une appropriation illicite ou carrément comme un geste meurtrier: comme si lui enlever le bijou entraînerait aussi sa vie alors que pour d'autres cela va de soi.

« Lorsque ma mère m'a transmis ses perles, elle m’a dit que ma grand-mère les avait toujours portées; que depuis qu'elle était morte juste avant ma naissance, elle les a toujours portées et que maintenant c'était à mon tour de les mettre. J'ai été très émue parce que maman dit que je suis la réincarnation de ma grand-mère, il paraît que j'ai le même caractère qu'elle. Et puis, j'ai pris sa succession, quoi ».

Cet exemple parmi d'autres montre bien comment le collier transmis correspond et instaure à la fois la place que le destinataire occupe dans la généalogie de la famille, ainsi que les liens privilégiés. Les prétendues ressemblances physiques et morales entre donateur et destinataire, les affections électives, ainsi que les règles coutumières et le rang de naissance sont autant de facteurs et de variantes qui conditionnent les modalités et les stratégies de la destination. Sans oublier les singularités de la vie qui peuvent perturber l'ordre des choses.

Barbara a aujourd'hui 45 ans, elle était très liée à sa tante paternelle, décédée à la suite d'un accident de voiture qui avait épargné par miracle la mère de Barbara. Cette 
Coffret à bijoux

Lieu de mémoire perpétuant aussi la mythologie familiale, à cet objet précieux par son contenu - et parfois en lui-même - s'accroche,

selon les mots de Pierre Michon, « toute la joaillerie verbale qui éclate dans les étranges noms propres des ä̈eux, dans la centième variante d'une histoire qu'on connaît, dans les motifs obscurs des mariages, des morts ».

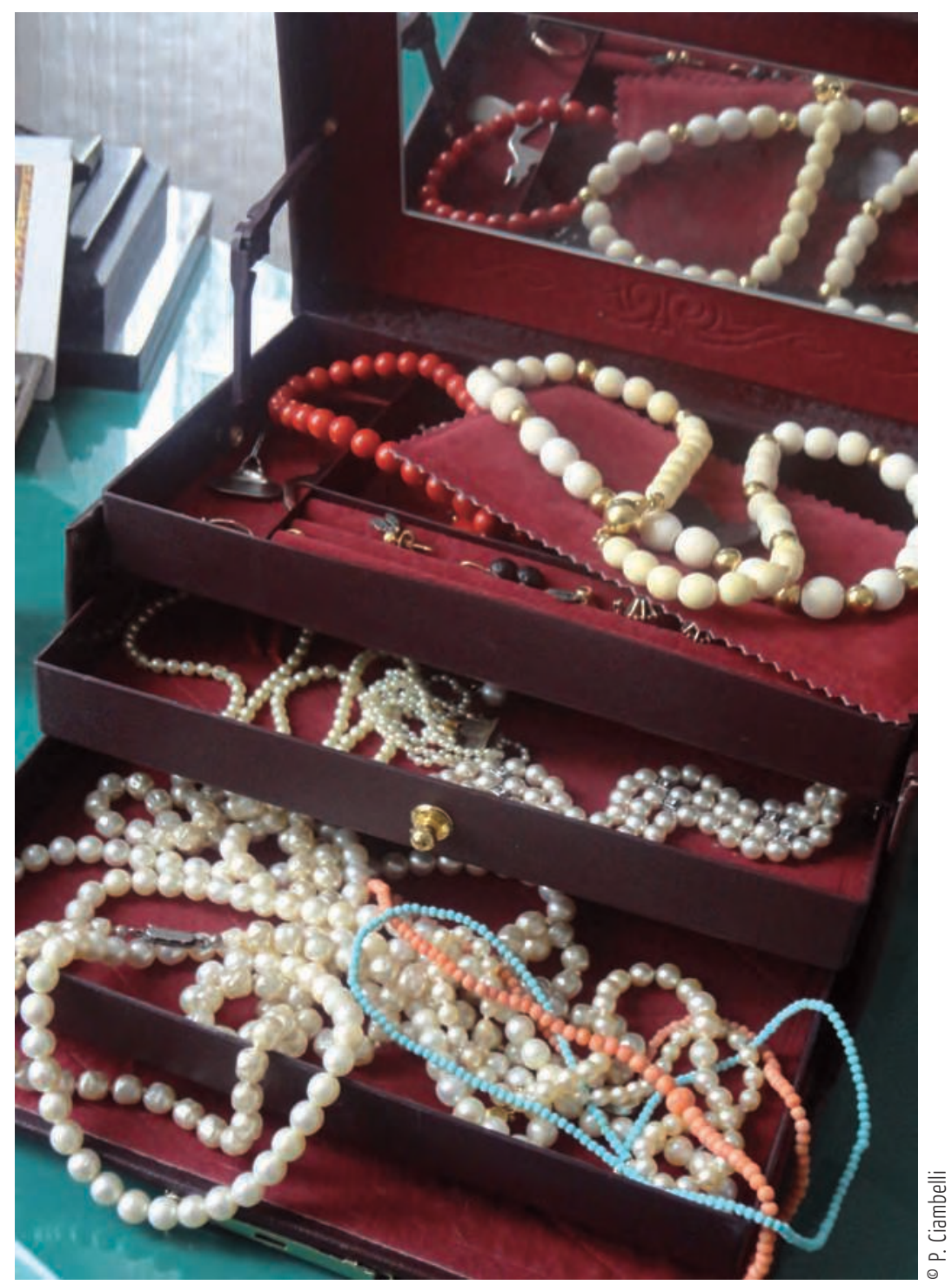

mort inattendue a suscité des conflits de famille pour la répartition d'un héritage sans testament. Affectée par cette perte soudaine et assombrie par l'acharnement de certains membres de la famille insensibles à ses yeux à toute valeur affective dans le partage des biens, Barbara a décidé de « faire disparaître » le collier de perles que sa tante lui avait destiné en secret de son vivant. Elle ne pouvait pas supporter qu'il soit « dispersé », pour elle, il représentait sa tante, il en était presque son émanation, le signe du lien privilégié qui la liait à elle. «Je ne pouvais pas la perdre deux fois». La «fausse perte» du collier de perles a permis à Barbara de rompre le pacte secret qui la liait à cette tante qui comptait «plus que sa mère » mais au prix d'un autre secret instauré vis-à-vis du reste de la famille, frappée par un geste d'usurpation qui a, de fait, mis le collier hors circulation ${ }^{23}$. 


\section{$\&$}

Hier déclinées à tous les temps, les perles - blanches ou noires, fines ou de culture, rondes ou baroques, d'eau douce ou d'eau salée - se conjuguent aujourd'hui à tous les modes au mépris des frontières spatiales et temporelles.

Outil esthétique avec ses codes et son langage, marque corporelle et sociale, objet " précieux », le collier de perles occupe une place privilégiée dès la prime enfance où il est au cœur des jeux d'apprentissage et d'initiation à la parure. De la toute petite première dent de lait, "perdue » et transformée en première "perle », à la paire enfilée dans les lobes percés de la jeune fille en fleurs avec le fil dégradé ouvrant au temps de la jeunesse jusqu'au collier à plusieurs rangs destiné à la femme accomplie, les perles ont conquis de nouveau une place privilégiée dans les coffrets à bijoux aux côtés d'autres parures délaissées dans le temps et réalisées, également, à partir de produits de la mer tels que le corail.

L'analogie entre la perle et le féminin se dessine autour de son origine « merveilleuse ", de sa marginalité dans l'univers aquatique, de l'acte même de son appropriation par les hommes ainsi qu'avec un vocabulaire qui rappelle les phases de la vie, de la naissance à la mort en passant par la croissance, la reproduction et le vieillissement. Sans oublier ses vertus et le contact corporel qui produit des façonnages et des échanges de propriétés parfois visibles dans la perte de brillance et d'éclat jusqu'au jaunissement qui trahit un âge de la vie où la « beauté » se trouve définitivement confisquée et préfigure ces larmes, frontière labile entre la gestation et la mort.

Objet d'accord ou de désaccord, de désir ou de déni, porté ou mis à l'écart, le collier de perles véhicule un destin et transmet une mémoire où s'inscrivent les événements importants et se cristallisent les liens les plus essentiels entre les femmes.

En demandant à mes interlocutrices de me parler de leurs colliers de perles - où et comment elles les ont acquis, quand elles les portent, ce qu'ils représentent - je les ai, de fait, invitées à raconter leur vie, à retrouver les événements heureux et malheureux, les amours et les ruptures qui s'inscrivent toujours, d'une manière ou d'une autre, dans le contenu d'un coffret à bijoux. Ce lieu de mémoire personnelle qui perpétue aussi, dans la mesure où il contient toujours quelque parure héritée ou transmise d'un ancêtre, l'histoire ou, plus exactement, comme le suggère très justement Pierre Michon (1984 : 25) la mythologie familiale. 


\section{NOTES}

Photo d'ouverture: Sur la commode. La négligence d'un geste fait que ce collier de perles trouve tout naturellement sa place et semble délivré par le gros coquillage rose pâle, le Strombus giga dessiné par Odile Redon en 1912 (carte postale, Le coquillage, pastel, Musée d'Orsay, Paris), P. Ciambelli.

1. Ce travail s'appuie sur des enquêtes réalisées dans la région toulousaine entre 2011 et 2012 auprès de bijoutiers, créateurs, femmes d'âge et statut social divers ainsi que sur un terrain précédent en Italie, France et Espagne entre 1993 et 1995 dans le cadre de l'appel d'offres de la Mission du Patrimoine Ethnologique: «Les jeux du paraître. Parure et esthétique du corps » et dont les résultats ont fait l'objet de publications (Albert-Llorca \& Ciambelli 1995, Ciambelli 1996 : 115-130, Albert-Llorca 1997 : 69-82, Ciambelli 2002).

2. Ce qui ne veut pas dire qu'elle fait l'unanimité. Certaines de mes interlocutrices sont catégoriques: elles les ont toujours détestées tant elles les associent aux stéréotypes sociaux de la fille bien rangée et de la dame de la bonne bourgeoisie dans lesquels on ne se reconnaît plus. Refus qui fait souvent office de rupture avec une tradition familiale dont la « gardienne » est une aieule conformiste et voulant imposer des règles de conduite strictes que des femmes modernes ont du mal à partager.

3. En plus de ses prétendues vertus de soigner et apaiser les «maux des femmes». L'usage de pierres brutes ou de gemmes polies, de cristaux et métaux pour des raisons thérapeutiques s'est banalisé tout comme le recours aux médecines alternatives et aux bienfaits des plantes.

4. Une quantité de marques s'est appropriée l'espace de la toile pour nourrir la culture de la perle, orienter les choix et surtout vendre à distance, pratique étonnante pour un bijou qui est en place depuis déjà un certain temps avec un bon succès commercial.

5. Juste à titre de rappel, dans le contexte chrétien, sainte Marguerite d'Antioche est invoquée par les femmes en couches alors que sainte Marguerite de Cortona en Italie protège les prostituées repenties.

6. Du latin pernula, diminutif de perna (« jambe », «cuisse de porc ») appliqué par ressemblance au coquillage connu sous le nom de pinne marine qu'on appelle aussi « jambonneau » (Rey 2000).

7. La place de la parure et notamment des matières aquatiques - perle et corail - dans une esthétique de la nudité et de l'altérité féminine a fait l'objet d'autres travaux qui se sont appuyés sur des corpus d'images picturales et gravées produites autour du XVI ${ }^{e}$ s. (Ciambelli 2008, 2009).

8. Tout comme dans le mythe d'origine du corail qu'Ovide décrit dans les Métamorphoses (L. IV, v. 740 et sqq.) et qui inspira le peintre Giorgio Vasari dans la représentation du tableau Persée délivrant Andromède (1570, Florence, Palazzo Vecchio).

9. Attraction qui fonde une allégorie spirituelle entre l'agate qui représente Jean et la perle le Christ. Tout comme pour les pierres précieuses, une notion physique se recouvre ainsi d'un propos métaphysique. Je renvoie pour cela ainsi que la perle comme métaphore de l'âme à Jean-Pierre Albert 1990 : 184-186.

10. Dictionnaire des Apocryphes II, col. 52 in Albert J.-P. $1990: 74$.

11. En Italie, dans certaines familles de la région de Forli, on atteste jusqu'au siècle dernier la pratique de poser dans la main d'un nouveau-né une bague «fleurie » d'une perle qui a la vertu de guérir « les perles »-dans le lexique médical, le nom vernaculaire de l'albugo ou tache blanche de la cornée - et des autres « maux des yeux ». (Tassoni 1973 : 291, 300, 332). Je ne peux ici qu'évoquer la complexité du lien entre la perle et le mauvais œil qui constitue l'objet d'une autre recherche laquelle met en perspective la perle et le corail. Je renvoie à mon analyse sur la relation entre le perçage des lobes, l'usage des boucles d'oreilles et la «vue » (Ciambelli 1996, 2002).

12. Il s'agit du conte type 480 , Les Fées d'après le titre de Ch. Perrault. On retrouve une variante dans Basile, « Le doie pizzelle » 1986 : 772-787.

13. Nature sensible et perméable aux changements de temps, à la lune et aux astres au moment de leurs « orages » biologiques (règles, grossesse, ménopause) comme l'a montré Yvonne Verdier (1979).

14. Dans quelques villages en Ariège et dans le Tarn a lieu encore le rituel du frottage des gencives avec la dent de Sainte Apollonie censée apaiser les douleurs et protéger des conséquences attribuées à la dentition telles les maux de ventre, les verminoses jusqu'aux convulsions.

15. On retrouve la relation entre les dents et les os comme substitut de la personne dans une pratique de la 
tradition juive, pour Again, la fête commémorative de la destruction du temple. « [...] nous, les enfants, on nous achetait une quantité de petites perles, vous savez, des perles minuscules. Avec ces toutes petites perles, je pouvais faire un petit poisson. C'était pour recueillir les os de tous les Juifs qu'on avait tués et qu'on avait éparpillés ». (Valensi \& Wachtel 1986 : 64).

16. Première collection, première richesse personnelle qui grandit et s'enrichit grâce aux gains de pièces plus ou moins convoitées ayant chacune leurs noms et leurs valeurs.

17. Sur la boîte à ouvrage v. W. Benjamin (2000 : 87). La boîte à boutons est la petite caisse des merveilles dans les souvenirs d'enfance de Lou Andreas Salomé (1980).

18. Le boulier est un autre jouet qui fascine autant que les billes et un cadeau incontournable de l'enfance; certains créateurs se sont inspirés de cet objet dynamique pour réaliser des pendentifs, des boucles d'oreilles et des bagues en insérant des perles mobiles, blanches et en couleur.

19. Chaque longueur a son appellation et désigne des styles, du plus rétro au plus provocateur en passant par le décontracté et l'opéra. La longueur princesse d'environ $45 \mathrm{~cm}$ est la plus classique: du collier de chien qui serre la gorge au sautoir, l'on passe d'environ $30 \mathrm{~cm}$ à plus d'un mètre.

20. Le perçage de la perle associée à l'onyx fait aussi l'objet d'un mythe d'origine fondé sur l'énigme posée par la reine de Saba au roi Salomon, résolue grâce à l'aide du termite et du ver à soie.
21. Un pouvoir mortifère qu'on retrouve dans l'expression « le chapelet se défile » lorsque les membres d'une famille disparaissent les uns après les autres et qui renvoie à son tour à la proximité entre l'usage religieux et l'usage profane du collier dans notre culture. Ce pouvoir funeste est aussi au cœur du récit La Perle de John Steinbeck et revient dans les écrits d'Albert Londres (1994) sur les pêcheurs de la mer Rouge et du golfe persique (lieux les plus fertiles jusqu'à l'introduction de la perle de culture) où les très recherchées « lou-lou » hantent comme des sirènes tous ceux qui y touchent même une seule fois et ceux qui la cherchent trop près des côtes.

22. Puisqu'ils ne doivent circuler que de manière verticale à la différence d'autres bijoux qui peuvent circuler horizontalement.

23. D'autres occasions peuvent être à l'origine de cette immobilisation qui s'apparente à une perte définitive, par exemple lorsqu'une femme lègue à la Vierge, la plus pure de toutes les femmes, son collier de perles en échange de l'obtention d'une grâce.

\section{RÉFÉRENCES}

Albert, J.-P. 1990 Odeurs de sainteté. La mythologie chrétienne des aromates. Paris: Éditions de l'EHESS.

Albert-Lorca, M. 1997 Linstant et l'éternité. Les bijoux dans la vie des femmes. Terrain 29: 69-82.

Albert-Llorca, M., Ciambelli, P. 1995 Parures des femmes, parures des Vierges. Rapport de recherche. Paris : Mission du Patrimoine Ethnologique.

Alexandre-Bidon, D. 1987 La dent et le corail ou La parure prophylactique de l'enfance au Moyen âge. In Razo $7:$ 5-35.

Andreas-Salomé, L. 1980 [1914] Du type féminin. In L’amour du narcissisme. Textes psychanalytiques. Paris: Gallimard (Connaissance de l'inconscient).

Basile, G.-B. [1634-1636] 1986 Lo cunto de li cunti. Milano: Garzanti.

Benjamin, W. 2000 Sens unique précédé de Enfance berlinoise. Paris: Éditions 10/18 (Domaine étranger).

Cardini, F. 1988 La conchiglia e la perla. Abstracta 26: 46-53. 
Chagall, B. 1994 Lumières allumées. Illustrations de Marc Chagall. Paris: Gallimard.

Choron-Baix, C. 2000 Transmettre et perpétuer aujourd'hui. Ethnologie Française « Envers et revers de la transmission $» 3: 357-360$.

Ciambelli, P. 2009 Le corps sauvage. In G. Vigarello (dir.) 100000 ans de beauté. Age classique/confrontations. Paris: Gallimard: 164-165.

— 2008 « Couleur sauvage. Images de l'altérité dans l'œuvre de Théodore de Bry ». In J.-P. Albert (dir. et al.) Coloris corpus. Paris: CNRS Éditions: 367-374.

- 2002 Bijoux à secrets. Paris: Éditions de la Maison des Sciences de l'homme (Ethnologie de la France).

— 1996 La Boucle et la Marque. Terrain « Amour » 27: 115-130.

Didi-Huberman, G. 1999 Ouvrir Venus. Nudité, rêve, cruauté. Paris: Gallimard (Le temps des images).

Londres, A. [1931] 1994 (rééd.) Pêcheurs de perles. Paris: Le Serpent à Plumes.

Malaguzzi, S. 2000 Perle. Firenze: Octavo, Officine grafiche De Agostini.

Michon, P. 1984 Vies minuscules. Paris: Gallimard (Folio).

Molière 1656 Le Dépit amoureux.

Ovide 1992 Les Métamorphoses. In J.-P. Néraudau (dir.), trad. G. Lafaye, Paris: Gallimard (Folio classique).

Perrault, C. 1697 Les fées. In Les contes de ma mère l'Oye.

Plinio 1983-1988 Storia naturale. Torino: Einaudi (Millenni).

Rey, A. (dir.) 2000 Dictionnaire historique de la langue française. Paris: Dictionnaires Le Robert.

Steinbeck, J. 1950 La Perle. Paris: Gallimard (Folio).

Tassoni, G. 1973 Arti e tradizioni popolari. Le inchieste napoleoniche sui costumi e le tradizioni nel regno italico. Bellinzona: Edizioni La Vesconta.

Terrain 1999 « Le Beau ». Terrain 32.

Turgeon, L. 2005 Les ceintures de wampum en Amérique. Communications 77: 17-37.

Valensi, L. \& Wachtel, N. (dir.) 1986 Mémoires juives. Paris: Gallimard (Archives).

Verdier, Y. 1979 Façons de dire, façons de faire. La laveuse, la couturière, la cuisinière. Paris: Gallimard. 


\section{RÉSUMÉ}

Colliers de perles. Transmission, circulation, mémoire du féminin. Lorsqu'on les nomme au pluriel, les perles désignent le collier, et sous cette forme, depuis fort longtemps sont emblématiques de l'univers féminin. Blanches ou noires, fines ou de culture, rondes ou baroques, d'eau douce ou d'eau salée, les perles aujourd'hui traversent les frontières et se conjuguent à tous les temps et à tous les modes. L'analogie entre cette matière-parure et le féminin se dessine autour de l'origine « merveilleuse » de la perle, de sa position dans l'univers aquatique, de l'acte même de son appropriation par les hommes ainsi que de son lexique. Objet « précieux » possédant ses codes et son langage, le collier de perles s'il est initiation à la parure dès l'enfance accompagne aussi des parcours de vie. À ce titre, il véhicule des destins, transmet des mémoires et inscrit les liens les plus essentiels entre les femmes.

\section{ABSTRACT}

Pearl necklaces. Feminine gender memory, transmission, circulation. When expressed in the plural, pearls designate the necklace, and have under such form been an emblem of the feminine universe for a very long time. "White" or "black", cultivated or natural, round or baroque, from fresh or salty waters, pearls today cross over borders, easily inflected in any time and mode. The analogy between this material/ornament and femininity develops around the "marvelous" origin of the pearl, its place in the aquatic world, the very act of its appropriation by men, and its vocabulary. "Precious" object having its own codes and language, the pearl necklace can be an initiation ornament since childhood as well as a companion along a life path. As such, it conveys destinies, transmits memories and defines most essential ties between women.

\section{MOTS CLÉS}

Perle, bijou, corps féminin, esthétique, destin

\section{KEYWORDS}

Pearl, jewel, feminine body, aesthetics, destiny 